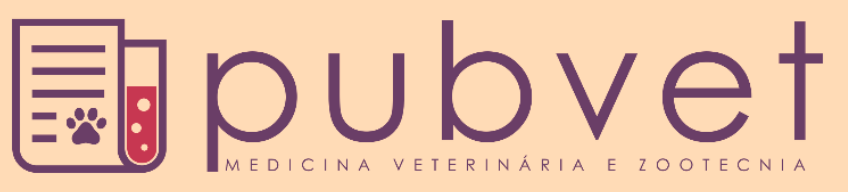

https://doi.org/10.31533/pubvet.v15n04a784.1-4

\title{
Deficiência de cobalto em bovinos: Revisão
}

\author{
Rodrigo Aquino Ventura', Gabriel Dantas Cuzzuol', Tayná Bolsam da Silva ${ }^{10}$, Ana Clara \\ Santana Malegoni ${ }^{1}$, Laiz Zaché Roque ${ }^{1 \Phi}$, Solimar Felipe Contarini de Oliveira ${ }^{19}$, Luiz Alexandre \\ $\operatorname{Moscon}^{2}{ }^{2}$, Diogo Almeida Rondon ${ }^{2} 9$, Clairton Marcolongo Pereira ${ }^{2, *} *$
}

${ }^{I}$ Graduandos de Medicina Veterinária, Centro Universitário do Espírito Santo - UNESC, Colatina - ES Brasil.

${ }^{2}$ Professor assistente, Laboratório de Patologia Veterinária, Faculdade de Medicina Veterinária, UNESC, Colatina-ES Brasil.

*Autor para correspondência, E-mail: clairton.marcolongo@terra.com.br

\begin{abstract}
Resumo. O Cobalto (Co) é um mineral relativamente raro e um elemento essencial necessário para a formação da vitamina $\mathrm{B}_{12}$ (cobalamina) em seres humanos e animais. A deficiência de cobalto é uma doença que só afeta ruminantes que ingerem em sua dieta baixas concentrações desse mineral, o que acarreta a deficiência dessa vitamina central em diversas vias metabólicas do organismo dos animais. Os objetivos desse trabalho foram fazer uma breve revisão sobre a deficiência de cobalto em ruminantes, abordando os aspectos epidemiológicos, as características clínicas-patológicas, o diagnóstico, o tratamento e o controle dessa enfermidade. A deficiência de cobalto é caracterizada clinicamente por inapetência, perda de peso e problemas reprodutivos. A doença ocorre em todos os biomas e regiões do Brasil, afetando principalmente animais criados em manejo extensivo. O diagnóstico presuntivo baseia-se no histórico e na observação dos rebanhos, nos quais os animais emagrecem apesar da pastagem abundante e o controle é feito pela suplementação com o mineral.
\end{abstract}

Palavras-chave: Cobalto, vitamina $\mathrm{B}_{12}$, deficiência, ruminante

\section{Cobalt deficiency in cattle: Review}

Abstract. Cobalt (Co) is a relatively rare mineral and an essential element necessary for the formation of vitamin B12 (cobalamin) in humans and animals. Cobalt deficiency is a disease that only affects ruminants that ingest in their diet low concentrations of this mineral, which leads to the deficiency of this central vitamin in several metabolic pathways in the animals' organisms. The objectives of this work were to make a brief review of cobalt deficiency in ruminants, addressing epidemiological aspects, clinical and pathological characteristics, diagnosis, treatment, and control of this disease. Cobalt deficiency is clinically characterized by inappetence, weight loss, and reproductive problems. The disease occurs in all biomes and regions of Brazil, affecting mainly animals raised in extensive management. The presumptive diagnosis is based on the history and observation of the herds, in which the animals lose weight despite the abundant pasture and the control is made by supplementation with the mineral.

Keywords: Cobalt, vitamin B12, deficiency, ruminant

\section{Deficiencia de cobalto em Bovinos: Revisión}

Resumen. El cobalto (Co) es un mineral relativamente raro y un elemento esencial necesario para la formación de vitamina B12 (cobalamina) en humanos y animales. La deficiencia de cobalto es una enfermedad que solo afecta a los rumiantes que ingieren en su dieta bajas concentraciones de este mineral, lo que conduce a la deficiencia de esta vitamina central en varias vías metabólicas en los organismos de los animales. Los 
objetivos de este trabajo fueron hacer una breve revisión de la deficiencia de cobalto en rumiantes, abordando aspectos epidemiológicos, características clínicas y patológicas, diagnóstico, tratamiento y control de esta enfermedad. La deficiencia de cobalto se caracteriza clínicamente por inapetencia, pérdida de peso y problemas reproductivos. La enfermedad se presenta en todos los biomas y regiones de Brasil, afectando principalmente a animales criados en manejo extensivo. El diagnóstico presuntivo se basa en la historia y observación de los rebaños, en los que los animales pierden peso a pesar de la abundancia de pastos y el control se realiza mediante suplementación con el mineral.

Palabras clave: Cobalto, vitamina B12, deficiencia, rumiante

\section{Introdução}

O Cobalto (Co) é um mineral relativamente raro e um elemento essencial necessário para a formação da vitamina $\mathrm{B}_{12}$ (cobalamina) em seres humanos e animais (Barceloux \& Barceloux, 1999). A deficiência de cobalto é uma doença que só afeta ruminantes que ingerem em sua dieta baixas concentrações desse mineral, o que acarreta a deficiência dessa vitamina central em diversas vias metabólicas do organismo dos animais. A enfermidade é caracterizada clinicamente por inapetência, perda de peso e problemas reprodutivos (Patterson, 2017).

De fato, as deficiências minerais podem causar graves prejuízos econômicos desencadeadas pela queda na produtividade e até mesmo podem se constituir um entrave à melhoria dos rebanhos (Tokarnia et al., 2010). Assim, os objetivos desse trabalho foram fazer uma breve revisão sobre a deficiência de cobalto em ruminantes, abordando os aspectos epidemiológicos, as características clínicas-patológicas, o diagnóstico, o tratamento e controle dessa enfermidade.

\section{Epidemiologia}

A deficiência de cobalto ocorre em todos os biomas e regiões do Brasil, sendo relatada em vários estados do país (Riet-Correa, 2007; Tokarnia et al., 2010). Estima-se que mais da metade dos animais acometidos pela doença são criados em manejo extensivo (Tokarnia et al., 2010). Os animais criados em confinamento recebem suplementação mineral e são alimentados com produtos que contém valores minerais e proteicos necessários para o bom funcionamento do organismo. Ao contrário, os animais criados em regime extensivo, não recebem suplementação e acabam apresentando a deficiência desse mineral (Riet-Correa, 2007).

As pastagens brasileiras são compostas principalmente de gramíneas, que para nutrição animal não possui teor energético que supra as necessidades do organismo sem a adição de suplementação de mineral aos animais (Riet-Correa, 2007). Algumas regiões, como a centro-oeste, a deficiência deste elemento em forrageiras é significativa, principalmente em condições de solo alcalino, interferindo na absorção de Co pelas plantas, além de excessos de manganês, que inibe a disponibilidade deste mineral pela planta (González et al., 2000).

A carência de Cobalto nas pastagens e nos rebanhos, foi descrita em uma diversidade de Estados, dentre eles: São Paulo, Minas Gerais, Mato Grosso, Santa Catarina, Roraima, Maranhão, além de diversas regiões do Espírito Santo (Tokarnia et al., 2010).

Pastos contendo menos de $0,07 \mathrm{mg} / \mathrm{kg}$ de matéria seca pode resultar em doença clínica em bovinos e ovinos (Patterson, 2017).

\section{Patogenia}

O cobalto é um mineral essencial na dieta de ruminantes porque esse elemento é armazenado no organismo animal em pequenas quantidades e em poucos tecidos. Nesses animais, a única função conhecida desse mineral é a síntese de vitamina $B_{12}$ no rúmen (Patterson, 2017). Nesse tecido, a vitamina $\mathrm{B}_{12}$ é utilizada como cofator na síntese de ácidos graxos voláteis, precursores de glicose (Smith, 2006). A deficiência de Co causará a inabilidade do ruminante em metabolizar ácido propiônico (González et al., 2000). Além disso, a hematopoiese e a gliconeogênese podem ser afetadas diretamente pela 
deficiência de vitamina $\mathrm{B}_{12}$, bem como o metabolismo de carboidratos, lipídeos e diversos outros mecanismos metabólicos dos animais (Smith, 2006).

\section{Sinais clínicos}

Não há sinal patognomônico na deficiência de cobalto, porém, pode-se observar perda gradativa do apetite, fraqueza, emagrecimento, mesmo em pastagem viçosa; lacrimação e mucosas pálidas. Em bovinos, pode-se observar a presença de Alotriofagia (pica) (Patterson, 2017). Além disso, a falta desse mineral no organismo pode causar engrossamento da pele e a alotriofagia, caracterizada principalmente pela ingestão de cascas de árvores e de madeira (Moraes, 2001; Riet-Correa, 2007).

\section{Patologia clínica}

Os animais com deficiência de cobalto apresentam anemia normocítica, normocrômica, mas geralmente os valores hematológicos estão dentro dos padrões de normalidade devido à hemoconcentração (Patterson, 2017). Além disso, os animais podem apresentar hipoglicemia (<60 mg de glicose/dL de plasma) e diminuição da fosfatase alcalina (Patterson, 2017).

\section{Achados de necropsia e alterações histológicas}

$\mathrm{Na}$ necropsia, pode-se observar emaciação intensa, fígado mais claro e levemente amarelado e a carcaça pálida. Histologicamente, há degeneração gordurosa hepática, possível atrofia do tecido hematopoético da medula óssea e em casos extremos, hemossiderose esplênica (Tokarnia et al., 2010).

\section{Diagnóstico}

O diagnóstico presuntivo baseia-se no histórico e na observação dos rebanhos, nos quais os animais emagrecem apesar da pastagem abundante. Além disso, a alotriofagia é bastante sugestiva (Tokarnia et al., 2010). O diagnóstico definitivo pode ser realizado pela administração de Co aos animais, que rapidamente se recuperam ou pela dosagem desse elemento no fígado (Tokarnia et al., 2010).

\section{Diagnóstico diferencial}

Deve-se incluir no diagnóstico diferencial de deficiência de Co em ruminantes a carência nutricional, como proteína e energia, parasitoses gastrintestinais, deficiência de cobre e a tuberculose (Patterson, 2017).

\section{Tratamento}

$\mathrm{O}$ tratamento pode ser realizado pela administração intramuscular de vitamina $\mathrm{B}_{12}$ ou por via oral de cobalto ou vitamina $B_{12}$, sendo esta última eficaz com doses de $0,1 \mathrm{mg} / \mathrm{kg}$, porém, devem ser realizadas doses maiores ou repetidas caso necessário (Patterson, 2017). Esta enfermidade pode, também, ser tratada pela administração de sal mineral na dieta dos animais contendo Co (Silva et al., 2017).

\section{Controle}

Um dos principais métodos de controle é suplementação gradativa de minerais ao rebanho, que é um fator imprescindível na nutrição animal. Coberturas das pastagens com sais minerais, uso de spray foliares, fertilização adequada do solo e alimentação com inclusão de fontes de Vitamina $\mathrm{B}_{12}$, estão inclusos na metodologia de controle da enfermidade, sendo um método ouro para prevenção (Patterson, 2017).

Para o controle e prevenção da deficiência de Co, pode ser feito a aplicação nas pastagens com fertilizantes que contenham Co. Além de proporcionar o elemento, essa adubação também favorece a produção nas leguminosas, sendo estimado de $200-500 \mathrm{~g} / \mathrm{ha}$ de sulfato de cobalto, entre 2 a 4 anos (Tokarnia et al., 2010).

Por se tratar basicamente de uma doença causada pela deficiência no solo, juntamente com a falha no manejo do pasto e do rebanho, é imprescindível reconsiderar as técnicas de manejo e realizar tratamentos adequados em questão do solo. Em regiões do Brasil, tanto quanto em diversas regiões do 
mundo que apresentam climatologia desregulada mantendo ambientes quentes e secos, solos argilosos ou arenosos e períodos chuvosos esporadicamente, o solo tende a ser pobre em cobalto e outros microelementos, como relatado. Isso ocorre, pois o cobalto se concentra em altas taxas nos solos úmidos e alcalinos, o que não corresponde com os solos do Brasil. Para isso, sugere-se corrigir as pastagens a cada 3 ou 4 anos com sulfato de cobalto a 1,5 kg/hectare (Smith, 2006).

\section{Agradecimentos}

À Fundação de Amparo à Pesquisa e inovação do Espírito Santo - FAPES e ao Centro Universitário do Espírito Santo - UNESC.

\section{Referências}

Barceloux, D. G., \& Barceloux, D. (1999). Cobalt. Journal of Toxicology: Clinical Toxicology, 37(2), 201-216. https://doi.org/10.1081/CLT-100102420

González, F., Barcellos, J., Patiño, H., \& Ribeiro, L. (2000). Perfil metabólico em ruminantes: seu uso em nutrição e doenças nutricionais. UFGRS, Porto Alegre, Brasil., 89.

Moraes, S. S. (2001). Principais deficiências minerais em bovinos de corte. Campo Grande, MS: Embrapa Gado de Corte, 2001.

Patterson, C. (2017). Veterinary Medicine: A Textbook of the Diseases of Cattle, Horses, Sheep, Pigs, and Goats, 11th edition, Volumes 1 and 2. The Canadian Veterinary Journal, 58(10), 1116. https://www.ncbi.nlm.nih.gov/pmc/articles/PMC5603921/

Riet-Correa, F. (2007). Doenças de ruminantes e eqüídeos. Santa Maria: Pallotti.

Silva, N. C. D., Martins, T. L. T., \& Borges, I. (2017). Efeito dos microminerais na alimentação de ruminantes. Ciência Animal, 27(1), 75-98.

Smith, M. O. (2006). Tratado de medicina interna de grandes animais (Vol. 1). Manole.

Tokarnia, C. H., Peixoto, P. V, Barbosa, J. D., Brito, M. F., \& Döbereiner, J. (2010). Deficiência de cobre. Deficiências Minerais Em Animais de Produção, 88-102.

Histórico do artigo:

Recebido: 24 de agosto de 2020.

Aprovado: 15 de outubro de 2020 .

Disponível online: 22 de janeiro de 2021.
Licenciamento: Este artigo é publicado na modalidade Acesso Aberto sob a licença Creative Commons Atribuição 4.0 (CC-BY 4.0), a qual permite uso irrestrito, distribuição, reprodução em qualquer meio, desde que o autor e a fonte sejam devidamente creditados. 